\title{
Spontaneous adenoid bleeding presenting as epistaxis and hematemesis
}

\begin{abstract}
The adenoid mass of lymphoid tissue is situated on the posterior wall of the nasopharynx $\&$ occupies much of that cavity in young children. Patient with adenoid hypertrophy will present with variety of symptoms including nasal obstruction \& mouth breathing, snoring, sleep apnea, recurrent pharyngeal infections, chest infections \& recurrent otitis media. Here we present a six years old girl presenting with hematemesis for one week and the complaint of periodic epistaxis. GI evaluation was preformed \& no obvious source of bleed was observed. After otolaryngology consultation, detailed history and nasopharyngoscopy revealed hypertrophied adenoids as the source of bleeding. Adenoidectomy was carried out \& seen to be curative after 12 months follow up. This paper presented our experience in the management of spontaneous adenoid bleeding. To our knowledge; this is the second time this issue is tackled.
\end{abstract}

Volume 8 Issue I - 2018

\author{
Ali H Almomen, Jawaher A Alzaid \\ KFSH University, Saudi Arabia
}

Correspondence: Jawaher A Alzaid, KFSH University, Saudi Arabia,Tel+96650070539I,Email special_jaz@hotmail.com

Received: February 07, 2018 | Published: February 16, 2018

\section{Case report}

An 19.8kilogram 6years old girl was referred to King Fahad Specialist Hospital (Dammam, Saudi Arabia) with the complaint of 4times vomiting of fresh blood \& abdominal pain. This hematemesis was developed after she has been treated with ibuprofen due to sudden onset of fever 38C. Several diagnostic procedures had been performed in search for gastrointestinal bleeding. Esophagogastroduodenoscopy was done and revealed normal -No bleeding spot or fresh blood seen in upper GI tract-, only fresh blood seen in oropharynx. There was no finding suggestive of celiac disease. The chest X-ray and all the laboratory findings were normal except for noticeable anemia. Laboratory: $\mathrm{CBC}: \mathrm{Hgb}=7.5$, PLT $=830, \mathrm{INR}=1.1$

After admission and evaluation in a pediatric center \& ruled out ulcer, maillary weises, the patient was referred to our ENT clinic for more evaluation. Full HEENT examination revealed normal. The main complaint was hematemesis. Detailed history revealed complaint of recurrent epistaxis 1-2times per month. Also, she had history of black colored stool but no change in urine. No significant family history of bleeding tendency. Under general anesthesia, nasal endoscopy done showing spontaneous nasopharyngeal bleeding (Figure 1) from the adenoid not controlled with suction cautery, then adenoidectomy done $\&$ hemostasis done by packing with xylomethazolin \& observation for 7 minutes then pack removed with no further bleeding. Patient extubated \& shifted to the ICU 1 day for observation. Post operative, the patient became symptom-free and had no more complaint of bleeding or any other complication during our 12 months follow up. Pathology of nasopharyngeal mass was reported to be non specific chronic inflammation. We believe that adenoid tissue was the source of the bleeding.

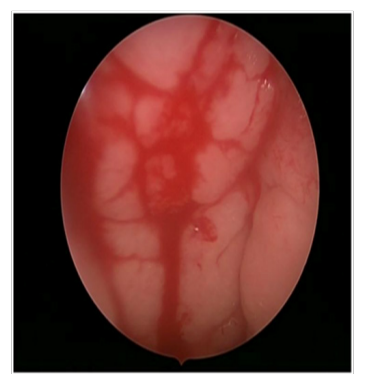

\section{Discussion}

Adenoids occupy much of nasopharynx cavity in young children. It is part of Waldeyer's ring which surrounds the entrance to the upper aero-digestive tract. Swallowed blood from a nose-bleed or intra-oral source can be mistaken for GI bleeding. ${ }^{1}$ Bleeding from GI tract is never normal and can signify serious disease or a common benign disorder. ${ }^{2}$ In some children - mainly up to the age of 5years- repeated upper respiratory tract infections cause adenoid enlargement. ${ }^{3}$ Patient with adenoid hypertrophy will present with nasal obstruction \& mouth breathing, snoring, sleep apnea, recurrent pharyngeal infections, chest infections \& recurrent otitis media. However, the physician should be aware of this presentation of adenoid hypertrophy or adenoiditis, which can be misinterpreted as upper GI bleeding and may impose some invasive diagnostic tests to the patient. ${ }^{4}$ A good view to adenoid can be seen with nasal endoscopy. It is accurate diagnostic tool in case of adenoid hypertrophy or adenoiditis or adenoid hemorrhage. Removal of the adenoids is particularly indicated when this disorder is accompanied by radiographic evidence of hypertrophy, especially following unsuccessful antimicrobial therapy. ${ }^{2}$ Adenoidectomy is seen to be curative in case of spontaneous adenoid bleeding where patient's main complaint disappear \& improves her life quality.

\section{Conclusion}

This paper presented our experience in the management of spontaneous adenoid bleeding case presenting with hematemesis \& epistaxis, to our knowledge, this is the second case of this issue. This case presentation reveals the importance of a full, detailed medical history and careful examination. Nasal endoscopy has significant role in diagnosis \& treatment of spontaneous adenoid bleeding during childhood, which should not be underestimated.

\section{Patient consent form}

Patient consent related case report take the permission from concerned patient.

\section{Acknowledgement}

None.

Figure I Nasal endoscopy shows spontaneous adenoid bleeding. 


\section{Conflict of interest}

The author declares no conflict of interest.

\section{References}

1. Baren Jill M. Pediatric emergency medicine. Elsevier Health Sciences; 2007
2. Essential of otolaryngology. 3rd ed. Frank E Lucente, Steven M Sobol.

3. Disease of the ear, nose and throat. 10th ed. Ray Clarke: Peter Bull; 2007.

4. Spontaneous adenoid hemorrhage mimicking upper gastrointestinal bleeding. Gholamreza Bayazian. 2017;7(1). 